\title{
Effect of Linseed Oil Supplementation on Hen Day Egg Production, Body Weight, Egg Shape Index, Economics and Egg Quality in Layers
}

\author{
Promila, Nand Kishore, Sajjan Sihag, Jyoti Shunthwal*, \\ Rakesh Verma and Saurabh Baloda
}
Department of Animal Nutrition, Lala Lajpat Rai University of Veterinary and Animal Sciences Hisar-125004, Haryana, India
*Corresponding author

\section{A B S T R A C T}

An experiment was conducted to find out the effect of supplementing different levels of linseed oil in the laying hens' diet on hen day egg production, body weight change, egg shape index and economics of feeding, during a period of 16 weeks. One hundred forty White Leghorn layers were randomly allocated into seven experimental groups having 5 replications of 4 birds in each and sited in individual cages from 22 to 38 weeks of age. The laying hens of control group $\left(\mathrm{T}_{1}\right)$ were fed a basal diet formulated as per BIS (2007)

\section{Keywords}

Hens, Linseed oil, Shape index, Economics, Omega-3 and hen day egg production.

Article Info

Accepted:

17 September 2017 Available Online: 10 November 2017 standards. The layers of treatment groups $\mathrm{T}_{2}, \mathrm{~T}_{3}, \mathrm{~T}_{4}, \mathrm{~T}_{5}, \mathrm{~T}_{6}$ and $\mathrm{T}_{7}$ were fed basal diet supplemented with linseed oil at levels of $1,2,2.5,3,3.5$ and $4 \%$, respectively. The results of the study unveiled that laying hen fed diets of $2.5 \%$ linseed oil $\left(\mathrm{T}_{4}\right)$ had $(\mathrm{P}<0.05)$ higher hen day egg production as compared to basal diet as well as the other dietary treatments. The results indicated a significant $(\mathrm{p}<0.05)$ increase in body weight in layers fed @ and $4 \%$ linseed oil as compared to control. Birds fed linseed oil had significant $(\mathrm{p}<0.05)$ increase in egg length and egg width in treatment group $\mathrm{T}_{5}, \mathrm{~T}_{6}$ and $\mathrm{T}_{7}$ as compared to control diet. But feeding of different levels of linseed oil in the diet of laying hens did not affect egg shape index. The results showed that feed cost value per dozen egg production decreased in treatment groups $\mathrm{T}_{4}(2.5 \%$ linseed oil) as compared to all other dietary treatments. Birds fed linseed oil had significant $(\mathrm{p}<0.05)$ decrease in palmitic $(\mathrm{C}: 16)$, stearic (C: 18) and oleic acid (18:1) being lowest in $\mathrm{T}_{7}$ (4\% linseed oil). Linoleic acid (C18:2) linolenic acid (18:3) and arachidonic acid (C20:4) significantly $(\mathrm{p}<0.05)$ increased being highest in $\mathrm{T}_{7}$ (4\% linseed oil) and lowest in $\mathrm{T}_{1}$ (control). Thus, from the present study it can be concluded that supplementation of linseed oil at different levels in laying hens' diet significantly $(\mathrm{P}<0.05)$, decrease average feed cost per dozen egg production and significantly $(\mathrm{P}<0.05)$ increase in hen day egg production, omega-3 fatty acid and body weight, whereas no effect of linseed oil supplementation on shape index.

\section{Introduction}

Indian poultry industry is one of the fastest growing segment of the agricultural sector today in India. As the production of agricultural crops has been rising at a rate of 1.5 to $2 \%$ per annum while the production of eggs and broilers has been rising at a rate of 8 to $10 \%$ per annum today India is world's fifth largest egg producer and the eighteenth largest producer of broiler. Driving this kind of expansion the contributing factors are 
growth in per capita, a growing urban population and falling poultry prices. A very significant feature of India's poultry industry is its transformation from a mere backyard activity into a major commercial activity in just about four decades which seems to be really fast. The kind of transformation has involved sizeable investments in breeding, hatching, rearing and processing. Indian farmers have moved from rearing nondescript birds to today's rearing hybrids and pure breeds

The Indian poultry industry has grown largely due to the initiative of private enterprise, minimal government intervention and very considerable indigenous poultry genetics capabilities and support from the complementary veterinary health, poultry feed, poultry equipments and poultry processing sectors. India is one of the few countries in the world that has put into place a sustained specific pathogen free (SPF) egg production project.

Linseed oil is highly unsaturated. It is rich in linolenic acid, which contains 3 double bonds with its first double bond 3 carbons from the terminal end (omega-3). The beneficial effects of consuming omega-3 fatty acids from fish include reducing heart disease, reducing circulating cholesterol levels and suppressing inflammation in humans (Klatt, 1986). This has prompted studies on the effect of feeding linseed oil or feedstuffs containing it to poultry as a means of increasing linolenic acid in eggs and poultry meat. As early as 1950, Chu and Kummerow reported that feeding a high level (25\%) of linseed oil to chickens caused increased linolenic acid in the fat of the skin and gizzard. Kummerow et al., (1948) also reported that feeding linseed oil to turkeys increased the iodine number of the fat and it was less stable to oxidation. Klose et al., (1952) showed that including 2\% of linseed oil in a turkey ration caused a large increase in the linolenic acid in the depot fat, a marked reduction in the induction period for fat oxidation and a marked fishy odor of the tissue. Egg shape index is defined as the ratio of width to length of the egg, and it is an important criterion in determining egg quality. Domestic hen eggs that are unusual in shape, such as those that are long and narrow, round, or flat-sided, cannot be placed in grade AA (nearly perfect) or A (slightly worse than AA) since an egg is generally oval in shape (72-76). Round eggs and unusually long eggs have poor appearances and do not fit well in egg cartons; therefore, they are much more likely to be broken during the shipment than the eggs of normal shape (Sarica and Erensayin, 2009).

\section{Materials and Methods}

A total of one hundred and forty single comb White Leghorn hens of commercial strain, 2223 weeks of age, in the first phase of their production cycle with an average weight of $1737 \pm 44.28 \mathrm{~g}$ were randomly divided in to seven treatment groups, having five replications with four birds in each replication. The laying hens of control group $\left(\mathrm{T}_{1}\right)$ were fed a basal diet formulated as per BIS (2007) standards, its ingredient and composition has been given in Table 1. The layers of treatment groups $\mathrm{T}_{2}, \mathrm{~T}_{3}, \mathrm{~T}_{4}, \mathrm{~T}_{5}, \mathrm{~T}_{6}$ and $\mathrm{T}_{7}$ were fed basal diet supplemented with linseed oil at levels of $1 \%, 2 \%, 2.5 \%, 3 \%$, $3.5 \%$ and $4 \%$, respectively. Hens were fed the experimental diet for sixteen weeks of experimental period beginning at 22 weeks of age and continued up to 38 weeks of age. The hens were offered feed and water ad libtum through linear feeder and waterers. Chemical composition (\%DM basis) and metabolizable energy $(\mathrm{Kcal} / \mathrm{Kg})$ of feed ingredients used in formulating the experimental diets and Composition and mixing rate of feed additives/ supplements and has been given in table 2 and 3 respectively. The data were 
analyzed using completely randomized design (Snedecor and Cochran, 1994).

\section{Analysis of feed ingredients}

Feed ingredients used in the diet formulations were analyzed for the proximate nutrients (AOAC, 2007). The chemical composition of different feed ingredients is presented in Table 2.

\section{Experimental diets}

The basal diet of laying hens was formulated as per BIS (2007) standards. The ingredient composition and chemical composition of the layers' control ration $\left(T_{1}\right)$, has been given in Table 3.

\section{Treatments}

$\mathrm{T}_{1}$ : Basal diet (Control) as per BIS (2007) specifications.

$\mathrm{T}_{2}$ : Basal diet+ $1 \%$ Linseed oil

$\mathrm{T}_{3}$ : Basal diet $+2 \%$ Linseed oil

$\mathrm{T}_{4}:$ Basal diet $+2.5 \%$ Linseed oil

$\mathrm{T}_{5}$ : Basal diet $+3.0 \%$ linseed oil

$\mathrm{T}_{6}$ : Basal diet $+3.5 \%$ linseed oil

$\mathrm{T}_{7}$ : Basal diet $+4.0 \%$ linseed oil

Feed additives and supplements were premixed and then mixed with weighed quantity of feed ingredients to make a homogenous mixture of rations.

\section{Percent hen day egg production}

Egg production were recorded daily, separate record for individual bird were maintained for entire experimental period i.e. 22-38 weeks of age of laying hens. Per cent hen day egg production was calculated by using following formula:

Total no. of eggs produced during the period Per cent hen day

Egg production $=-\times 100$

Total no. of hen days during the period

\section{Shape index}

The width and length of each egg was taken using Vernier caliper. Shape index was calculated as per the formula.

Shape index $=\frac{\text { Maximum width of egg }}{\text { Maximum length of egg }} \times 100$

Feed cost per dozen of egg production

Average feed cost per dozen of egg was calculated from the amount of feed (in $\mathrm{Kg}$ ) consumed during the period multiply by cost of per $\mathrm{kg}$ feed.

\section{Fatty acid profile}

For fatty acid profile the yolks from three eggs were separated for each replicate, pooled, homogenized and fat separation by the method of Angelo et al., (1987). Methyl ester was prepared by the method of Luddy $e t$ al., (1968) and then fractionation of methyl ester by using gas chromatograpy.

\section{Results and Discussion}

\section{Hen day egg production}

The percent hen day egg production, for the 7 periods (22-24, 24-26, 26-28, 28-30, 30-32, 32-34, 34-36 and 36-38 weeks of age) of 2 weeks each and cumulative production of 1-8 periods (22-38weeks), are presented in Table 5 . 
The cumulative hen day egg production values were $60.69,58.25,63.78,69.90,61.08$, 60.62 and 58.29 percent in treatment groups $\mathrm{T}_{1}, \mathrm{~T}_{2}, \mathrm{~T}_{3}, \mathrm{~T}_{4}, \mathrm{~T}_{5}, \mathrm{~T}_{6}$ and $\mathrm{T}_{7}$, respectively. The results of the study unveiled that laying hen fed diets of $2.5 \%$ linseed oil $\left(\mathrm{T}_{4}\right)$ had $(\mathrm{P}<0.05)$ higher hen day egg production as compared to that of hens fed maize based basal diet as well as the other treatment. Similarly the results of study (during 22-24weeks) indicated that, when diets of layers were supplemented with $2.5 \%$ of the linseed oil there was a significant $(\mathrm{P}<0.05)$ positive effect on per cent hen day egg production in comparison to control group and other levels of linseed oil.

The similar trends of hen day egg production were found during 28-30 and 30-32 weeks of age of laying hens. The minimum hen day egg production (ranged from $50.35 \%$ in $\mathrm{T}_{7}$ to $52.07 \%$ in $\mathrm{T}_{1}$ ) was found at age of 36-38 weeks of age and maximum $\left(75.71 \%\right.$ in $\left.\mathrm{T}_{4}\right)$ at 22-24 weeks of age in different dietary treatments.

In nutshell, the data of the study revealed that feeding of hens with $2.5 \%$ linseed oil had significantly $(\mathrm{P}<0.05)$ higher percent hen day egg production, followed by hens fed with $2 \%$ linseed oil compared to control group and other dietary treatments, however, treatment groups $\mathrm{T}_{1}, \mathrm{~T}_{2}, \mathrm{~T}_{5}, \mathrm{~T}_{6}$ and $\mathrm{T}_{7}$ did not show any significant $\quad(\mathrm{P}<0.05) \quad$ difference among themselves. These finding are in agreement with the findings of Beynen (2004), Celebi and Utlu (2006), (Augustyn et al., 2006) and Aziza et al., (2013). On contrary, Van Elswyk (1997 b), Ansari et al., (2006) and Švedová et al., (2008) reported that linseed oil supplementation in ration of layer decreases egg production.

Galobart et al., (2001) reported no influence of feeding $5 \%$ linseed oil on the egg production of hens. Grobas et al., (2001) found that the hens fed 5 or $10 \%$ linseed oil produced similar number of eggs when compared to the hens without supplementation during a period of 12 weeks. Novak and Scheideler (2001) reported that egg production was not significantly different $(\mathrm{P}<0.05)$ for the hens fed $10 \%$ flax seed compared to those on the diet with $0 \%$ flaxseed.

\section{Body weight changes}

The mean body weights of the experimental laying hens at the beginning of the experiment and at the end of the experiment are presented in Table 6. The results of the study depicted that all the experimental birds under different dietary treatments were in positive weights.

The collective mean values (22-38 weeks) of body weight gain of layers were $0.15,0.17$, $0.20,0.26,0.19,0.23$ and $0.32 \mathrm{~kg}$ in treatment groups $\mathrm{T}_{1}, \mathrm{~T}_{2}, \mathrm{~T}_{3}, \mathrm{~T}_{4}, \mathrm{~T}_{5}, \mathrm{~T}_{6}$ and $\mathrm{T}_{7}$, respectively. The statistical analysis of the data revealed that significant effect on the body weight gain of hens was observed by dietary supplementation of basal ration with different levels of linseed oil as compared to the non-supplemented control diet. Body weight gain was maximum in $\mathrm{T}_{7}$ and minimum in $\mathrm{T}_{1}$.

The results of the study depicted that all the experimental birds under different dietary treatments were in positive weights. The statistical analysis of the data revealed that significant effect on the body weight gain of hens was observed by dietary supplementation of basal ration with different levels of linseed oil as compared to the no added control diet. More the level of supplementation more was the gain.

This might be due to higher content of rations supplemented with linseed oil. Although, no literature was present in context of layer's growth performance. 
Table.1 Ingredient and chemical composition of ration for layers of control group

\begin{tabular}{|l|c|}
\hline Feed ingredients & Percentage \\
\hline Maize & 50 \\
\hline Groundnut cake & 7 \\
\hline Soybean Meal & 13 \\
\hline DORP & 12 \\
\hline Rice Polish & 5 \\
\hline Fish Meal & 6 \\
\hline Mineral Mixture & 3 \\
\hline Salt & 0.5 \\
\hline Shell Grit & 3.5 \\
\hline Chemical composition & \%DM basis \\
\hline CP & 19.04 \\
\hline CF & 6.74 \\
\hline EE & 3.61 \\
\hline NFE & 62.81 \\
\hline Ash & 7.80 \\
\hline Metabolizable energy*(Kcal/Kg) & 2697.17 \\
\hline
\end{tabular}

* calculated value (BIS, 2007), Feed additive included Spectromix-10g (Each g contained vitamin A- 82,500 IU, vitamin $\mathrm{D}_{3}$ 12,000 IU, vitamin B2- 50mg, and vitamin K- 10mg.), Spectrimix-BE-10g (Each g contained vitamin B1-80mg, vitamin $B_{6}-16 \mathrm{mg}$, Niacin- 120mg, vitamin $B_{12}-80 \mathrm{mg}$, Calcium Pantothenate- 80mg, vitamin E -160mg, L-lysine $\mathrm{HCl}-10 \mathrm{mg}$, DL-Methionine -10mg, and Calcium- 260mg) per $100 \mathrm{Kg}$ of ration.

Table.2 Chemical composition (\%DM basis) and metabolizable energy (Kcal/Kg) of feed ingredients used in formulating the experimental diets

\begin{tabular}{|c|c|c|c|c|c|c|}
\hline Ingredients & CP & CF & EE & Ash & ME* & Cost/100kg \\
\hline Maize & 9 & 2 & 4 & 1.5 & 3300 & 1719 \\
\hline GNC & 44 & 10 & 1 & 8 & 2400 & 2959 \\
\hline Soyabean meal & 44 & 6.5 & 0.8 & 6 & 2250 & 3643 \\
\hline Rice polish & 12.7 & 5 & 14 & 8 & 2700 & 1287 \\
\hline DORP & 16 & 14 & 0.5 & 12.5 & 1800 & 894 \\
\hline Fish meal & 45 & 1 & 7 & 22 & 2180 & 5373 \\
\hline
\end{tabular}

* calculated value (BIS, 2007)

Table.3 Composition and mixing rate of feed additives/ supplements

\begin{tabular}{|c|c|c|}
\hline Additives/suppliments & Composition & $\begin{array}{l}\text { Mixing } \\
\text { rate/qtl }\end{array}$ \\
\hline Spectromix Powder & $\begin{array}{l}\text { Each g contained vitamin } \mathrm{A}-82,500 \mathrm{IU} \text {, vitamin } \mathrm{D}_{3}-12,000 \mathrm{IU} \text {, } \\
\text { vitamin B2- } 50 \mathrm{mg} \text {, and vitamin K- } 10 \mathrm{mg} \text {. }\end{array}$ & $10 \mathrm{~g} /$ quintal \\
\hline Spectromix-BE powder & $\begin{array}{l}\text { Each g contained vitamin B1- } 80 \mathrm{mg} \text {, vitamin } \mathrm{B}_{6}-16 \mathrm{mg} \text {, Niacin- } \\
120 \mathrm{mg} \text {, vitamin } \mathrm{B}_{12^{-}}-80 \mathrm{mg} \text {, Calcium Pantothenate- } 80 \mathrm{mg} \text {, vitamin } \\
\mathrm{E}-160 \mathrm{mg} \text {, L-lysine HCl- } 10 \mathrm{mg} \text {, DL-Methionine }-10 \mathrm{mg} \text {, and } \\
\text { Calcium- } 260 \mathrm{mg}\end{array}$ & $10 \mathrm{~g} /$ quintal \\
\hline Mineral mixture & $\begin{array}{l}\text { Mineral mixture for poultry: composition (w/w): moisture- } 3 \% \\
\text { (maximum), Calcium- } 32 \% \text { (minimum), Phosphorus- } 6 \% \\
\text { (minimum), Manganese- } 0.27 \% \text { (minimum), Iodine- } 0.01 \% \\
\text { (minimum), Zinc- } 0.26 \% \quad \text { (minimum), Fluorine- } 0.03 \% \\
\text { (maximum), Copper- } 0.001 \% \text { (minimum) and Iron- } 0.001 \% \\
\text { (minimum). }\end{array}$ & $3 \mathrm{Kg} /$ quintal \\
\hline
\end{tabular}


Table.4 Chemical composition of ration for layers of different treatment groups

\begin{tabular}{|l|l|l|l|l|l|l|l|}
\hline \multirow{2}{*}{$\begin{array}{l}\text { Chemical } \\
\text { composition }\end{array}$} & \multicolumn{2}{l}{ \% DM basis } \\
\cline { 2 - 8 } & $\mathbf{T 1}$ & $\mathbf{T 2}$ & $\mathbf{T 3}$ & $\mathbf{T 4}$ & $\mathbf{T 5}$ & $\mathbf{T 6}$ & $\mathbf{T 7}$ \\
\hline CP & 19.04 & 19.10 & 19.07 & 19.06 & 19.08 & 19.05 & 19.03 \\
\hline CF & 6.74 & 6.23 & 6.16 & 6.03 & 5.78 & 5.66 & 5.47 \\
\hline EE & 3.61 & 4.25 & 4.67 & 5.34 & 5.73 & 6.19 & 7.05 \\
\hline Ash & 7.80 & 8.04 & 7.93 & 8.11 & 8.02 & 7.89 & 7.96 \\
\hline NFE & 62.81 & 62.38 & 62.17 & 61.39 & 62.58 & 61.21 & 60.49 \\
\hline ME* Kcal/Kg & 2697.17 & 2757.59 & 2816.83 & 2846.01 & 2874.92 & 2903.54 & 2931.89 \\
\hline
\end{tabular}

* calculated value

Table.5 Mean values of percent hen day egg production during progressive age (weeks) under different dietary treatments

\begin{tabular}{|l|l|l|l|l|l|l|l|l|}
\hline $\begin{array}{l}\text { Weeks/ } \\
\text { Treatment }\end{array}$ & $\mathbf{T}_{\mathbf{1}}$ & $\mathbf{T}_{\mathbf{2}}$ & $\mathbf{T}_{\mathbf{3}}$ & $\mathbf{T}_{\mathbf{4}}$ & $\mathbf{T}_{\mathbf{5}}$ & $\mathbf{T}_{\mathbf{6}}$ & $\mathbf{T}_{\mathbf{7}}$ & $\mathbf{C D}$ \\
\hline \multirow{2}{*}{$22-24$} & $62.44^{\mathrm{b}}$ & $61.78^{\mathrm{b}}$ & $65.0^{\mathrm{ab}}$ & $75.71^{\mathrm{a}}$ & $61.88^{\mathrm{b}}$ & $67.85^{\mathrm{ab}}$ & $60.30^{\mathrm{b}}$ & \multirow{2}{*}{11.39} \\
& \pm 1.66 & \pm 4.61 & \pm 5.07 & \pm 2.16 & \pm 5.26 & \pm 4.11 & \pm 3.07 & \\
\hline \multirow{2}{*}{$24-26$} & $73.92^{\mathrm{a}}$ & $62.50^{\mathrm{b}}$ & $72.50^{\mathrm{ab}}$ & $74.28^{\mathrm{a}}$ & $71.42^{\mathrm{ab}}$ & $67.85^{\mathrm{ab}}$ & $67.50^{\mathrm{ab}}$ & 10.94 \\
& \pm 1.75 & \pm 3.95 & \pm 5.58 & \pm 2.80 & \pm 5.21 & \pm 1.60 & \pm 3.54 & \\
\hline \multirow{2}{*}{$26-28$} & $61.78^{\mathrm{ab}}$ & $51.42^{\mathrm{b}}$ & $69.64^{\mathrm{a}}$ & $65.35^{\mathrm{a}}$ & $63.21^{\mathrm{ab}}$ & $56.78^{\mathrm{ab}}$ & $64.64^{\mathrm{a}}$ & \multirow{2}{*}{12.97} \\
& \pm 6.84 & \pm 4.13 & \pm 4.66 & \pm 2.44 & \pm 4.47 & \pm 1.91 & \pm 5.03 & \\
\hline \multirow{2}{*}{$28-30$} & $58.92^{\mathrm{b}}$ & $56.07^{\mathrm{b}}$ & $57.50^{\mathrm{b}}$ & $69.92^{\mathrm{a}}$ & $59.64^{\mathrm{ab}}$ & $60.00^{\mathrm{ab}}$ & $62.14^{\mathrm{ab}}$ & \multirow{2}{*}{10.91} \\
\hline \multirow{2}{*}{$30-32$} & \pm 5.11 & \pm 2.01 & \pm 3.50 & \pm 5.24 & \pm 4.32 & \pm 1.66 & \pm 2.85 & \\
& $60.00^{\mathrm{b}}$ & $59.28^{\mathrm{b}}$ & $59.28^{\mathrm{b}}$ & $70.35^{\mathrm{a}}$ & $58.21^{\mathrm{b}}$ & $57.14^{\mathrm{b}}$ & $55.00^{\mathrm{b}}$ & \multirow{2}{*}{8.15} \\
\hline \multirow{2}{*}{$32-34$} & \pm 2.37 & \pm 2.14 & \pm 4.57 & \pm 2.86 & \pm 1.21 & \pm 3.19 & \pm 2.14 & \\
& $58.57^{\mathrm{b}}$ & $59.64^{\mathrm{b}}$ & $67.14^{\mathrm{a}}$ & $70.71^{\mathrm{a}}$ & $58.92^{\mathrm{b}}$ & $59.64^{\mathrm{b}}$ & $53.56^{\mathrm{b}}$ & \multirow{2}{*}{6.50} \\
\hline \multirow{2}{*}{$34-36$} & \pm 1.73 & \pm 2.56 & \pm 2.37 & \pm 1.92 & \pm 2.39 & \pm 2.43 & \pm 2.19 & \\
\hline \multirow{2}{*}{$36-38$} & $57.85^{\mathrm{bc}}$ & $61.42^{\mathrm{b}}$ & $61.42^{\mathrm{b}}$ & $69.64^{\mathrm{a}}$ & $61.07^{\mathrm{b}}$ & $62.14^{\mathrm{b}}$ & $52.85^{\mathrm{c}}$ & \multirow{2}{*}{6.77} \\
& \pm 2.80 & \pm 2.68 & \pm 2.68 & \pm 1.13 & \pm 3.50 & \pm 1.99 & \pm 1.21 & \\
\hline \multirow{2}{*}{ Mean } & $52.07^{\mathrm{bc}}$ & $53.93^{\mathrm{bc}}$ & $57.78^{\mathrm{ab}}$ & $63.21^{\mathrm{a}}$ & $54.28^{\mathrm{bc}}$ & $53.57^{\mathrm{bc}}$ & $50.35^{\mathrm{c}}$ & \multirow{2}{*}{6.23} \\
& \pm 2.88 & \pm 1.82 & \pm 2.01 & \pm 2.08 & \pm 1.34 & \pm 3.04 & \pm 1.19 & \\
\hline
\end{tabular}

The mean values in same row with different superscripts differ significantly $(\mathrm{P}<0.05)$

Table.6 Body Weight changes $(\mathrm{Kg})$ of layers during the experimental period under different dietary treatments

\begin{tabular}{|l|c|c|c|}
\hline Treatments & Initial Body Weight & Final Body Weight & Body Weight gain(g) \\
\hline $\mathrm{T}_{1}$ & $1.78^{\mathrm{a}} \pm 0.05$ & $1.94 \pm 0.05$ & $0.15^{\mathrm{d}} \pm 0.01$ \\
\hline $\mathrm{T}_{2}$ & $1.78^{\mathrm{a}} \pm 0.06$ & $1.95 \pm 0.06$ & $0.17^{\mathrm{cd}} \pm 0.02$ \\
\hline $\mathrm{T}_{3}$ & $1.81^{\mathrm{a}} \pm 0.06$ & $2.01 \pm 0.05$ & $0.20^{\mathrm{cd}} \pm 0.03$ \\
\hline $\mathrm{T}_{4}$ & $1.75^{\mathrm{ab}} \pm 0.04$ & $2.01 \pm 0.05$ & $0.26^{\mathrm{ab}} \pm 0.02$ \\
\hline $\mathrm{T}_{5}$ & $1.77^{\mathrm{a}} \pm 0.04$ & $1.95 \pm 0.05$ & $0.19^{\mathrm{cd}} \pm 0.02$ \\
\hline $\mathrm{T}_{6}$ & $1.67^{\mathrm{ab}} \pm 0.04$ & $1.89 \pm 0.04$ & $0.23^{\mathrm{bc}} \pm 0.02$ \\
\hline $\mathrm{T}_{7}$ & $1.62^{\mathrm{b}} \pm 0.06$ & $1.94 \pm 0.05$ & $0.32^{\mathrm{a}} \pm 0.05$ \\
\hline $\mathrm{CD}$ & 0.14 & NS & 0.07 \\
\hline \multicolumn{2}{|c|}{} & \multicolumn{2}{c}{} \\
\hline
\end{tabular}


Table.7 Mean values of egg width (cm) during progressive age (weeks) under different dietary treatments

\begin{tabular}{|l|c|c|c|c|c|c|c|c|}
\hline $\begin{array}{l}\text { Weeks/ } \\
\text { Treatment }\end{array}$ & $\mathbf{T}_{\mathbf{1}}$ & $\mathbf{T}_{\mathbf{2}}$ & $\mathbf{T}_{\mathbf{3}}$ & $\mathbf{T}_{\mathbf{4}}$ & $\mathbf{T}_{\mathbf{5}}$ & $\mathbf{T}_{\mathbf{6}}$ & $\mathbf{T}_{\mathbf{7}}$ & $\mathbf{C} \mathbf{C}$ \\
\hline \multirow{2}{*}{$22-24$} & $3.79^{\mathrm{b}}$ & $3.88^{\mathrm{ab}}$ & $3.95^{\mathrm{a}}$ & $3.97^{\mathrm{a}}$ & $3.97^{\mathrm{a}}$ & $3.93^{\mathrm{ab}}$ & $4.00^{\mathrm{a}}$ & \multirow{2}{*}{0.14} \\
& \pm 0.05 & \pm 0.03 & \pm 0.02 & \pm 0.07 & \pm 0.04 & \pm 0.04 & \pm 0.07 & \\
\hline \multirow{2}{*}{$24-26$} & 4.06 & 4.07 & 4.16 & 4.06 & 4.13 & 4.18 & 4.16 & \multirow{2}{*}{$\mathrm{NS}$} \\
& \pm 0.02 & \pm 0.02 & \pm 0.03 & \pm 0.02 & \pm 0.04 & \pm 0.07 & \pm 0.08 & \\
\hline \multirow{2}{*}{$26-28$} & $4.11^{\mathrm{b}}$ & $4.15^{\mathrm{ab}}$ & $4.22^{\mathrm{ab}}$ & $4.11^{\mathrm{b}}$ & $4.25^{\mathrm{ab}}$ & $4.27^{\mathrm{ab}}$ & $4.31^{\mathrm{a}}$ & \multirow{2}{*}{0.19} \\
& \pm 0.03 & \pm 0.02 & \pm 0.04 & \pm 0.02 & \pm 0.10 & \pm 0.06 & \pm 0.12 & \\
\hline \multirow{2}{*}{$28-30$} & $3.97^{\mathrm{b}}$ & $4.13^{\mathrm{ab}}$ & $4.15^{\mathrm{a}}$ & $4.16^{\mathrm{a}}$ & $4.20^{\mathrm{a}}$ & $4.28^{\mathrm{a}}$ & $4.24^{\mathrm{a}}$ & \multirow{2}{*}{0.16} \\
& \pm 0.06 & \pm 0.03 & \pm 0.02 & \pm 0.06 & \pm 0.04 & \pm 0.04 & \pm 0.11 & \\
\hline \multirow{2}{*}{$30-32$} & 4.14 & 4.20 & 4.18 & 4.17 & 4.20 & 4.23 & 4.22 & \multirow{2}{*}{$\mathrm{NS}$} \\
& \pm 0.03 & \pm 0.05 & \pm 0.05 & \pm 0.02 & \pm 0.04 & \pm 0.02 & \pm 0.03 & \\
\hline \multirow{2}{*}{$32-34$} & $4.13^{\mathrm{b}}$ & $4.14^{\mathrm{b}}$ & $4.24^{\mathrm{ab}}$ & $4.13^{\mathrm{b}}$ & $4.22^{\mathrm{ab}}$ & $4.27^{\mathrm{ab}}$ & $4.35^{\mathrm{a}}$ & \multirow{2}{*}{0.14} \\
& \pm 0.04 & \pm 0.02 & \pm 0.04 & \pm 0.08 & \pm 0.03 & \pm 0.06 & \pm 0.04 & \\
\hline \multirow{2}{*}{$34-36$} & $4.04^{\mathrm{b}}$ & $4.13^{\mathrm{ab}}$ & $4.22^{\mathrm{a}}$ & $4.03^{\mathrm{b}}$ & $4.19^{\mathrm{ab}}$ & $4.28^{\mathrm{a}}$ & $4.23^{\mathrm{a}}$ & \multirow{2}{*}{0.16} \\
& \pm 0.05 & \pm 0.06 & \pm 0.01 & \pm 0.02 & \pm 0.04 & \pm 0.11 & \pm 0.05 & \\
\hline \multirow{2}{*}{$36-38$} & 4.08 & 4.08 & 4.10 & 4.18 & 4.24 & 4.19 & 4.22 & \multirow{2}{*}{$\mathrm{NS}$} \\
\hline \multirow{2}{*}{ Mean } & \pm 0.06 & \pm 0.06 & \pm 0.02 & \pm 0.04 & \pm 0.04 & \pm 0.12 & \pm 0.09 & \\
& $4.04^{\mathrm{d}}$ & $4.10^{\mathrm{cd}}$ & $4.15^{\mathrm{bc}}$ & $4.10^{\mathrm{cd}}$ & $4.17^{\mathrm{ab}}$ & $4.20^{\mathrm{ab}}$ & $4.22^{\mathrm{a}}$ & \multirow{2}{*}{0.06} \\
& \pm 0.02 & \pm 0.02 & \pm 0.02 & \pm 0.02 & \pm 0.02 & \pm 0.03 & \pm 0.03 & \\
\hline
\end{tabular}

The mean values in same row with different superscripts differ significantly $(\mathrm{P}<0.05)$

Table.8 Mean values of egg length $(\mathrm{cm})$ during progressive age (weeks) under different dietary treatments

\begin{tabular}{|l|l|l|l|l|l|l|l|l|}
\hline $\begin{array}{l}\text { Weeks/ } \\
\text { Treatment }\end{array}$ & $\mathbf{T}_{\mathbf{1}}$ & $\mathbf{T}_{\mathbf{2}}$ & $\mathbf{T}_{\mathbf{3}}$ & $\mathbf{T}_{\mathbf{4}}$ & $\mathbf{T}_{\mathbf{5}}$ & $\mathbf{T}_{\mathbf{6}}$ & $\mathbf{T}_{\mathbf{7}}$ & $\mathbf{C D}$ \\
\hline \multirow{2}{*}{$22-24$} & $5.43^{\mathrm{ab}}$ & $5.28^{\mathrm{b}}$ & $5.46^{\mathrm{ab}}$ & $5.32^{\mathrm{ab}}$ & $5.56^{\mathrm{a}}$ & $5.55^{\mathrm{a}}$ & $5.49^{\mathrm{ab}}$ & 0.24 \\
& \pm 0.05 & \pm 0.03 & \pm 0.02 & \pm 0.11 & \pm 0.11 & \pm 0.14 & \pm 0.03 & \\
\hline \multirow{2}{*}{$24-26$} & $5.57^{\mathrm{b}}$ & $5.77^{\mathrm{ab}}$ & $5.75^{\mathrm{ab}}$ & $5.66^{\mathrm{ab}}$ & $5.73^{\mathrm{ab}}$ & $5.87^{\mathrm{a}}$ & $5.82^{\mathrm{a}}$ & \multirow{2}{*}{0.22} \\
& \pm 0.10 & \pm 0.07 & \pm 0.07 & \pm 0.04 & \pm 0.06 & \pm 0.07 & \pm 0.11 & \\
\hline \multirow{2}{*}{$26-28$} & $5.62^{\mathrm{b}}$ & $5.72^{\mathrm{ab}}$ & $5.78^{\mathrm{ab}}$ & $5.67^{\mathrm{ab}}$ & $5.76^{\mathrm{ab}}$ & $5.85^{\mathrm{a}}$ & $5.86^{\mathrm{a}}$ & \multirow{2}{*}{0.21} \\
& \pm 0.09 & \pm 0.09 & \pm 0.05 & \pm 0.10 & \pm 0.05 & \pm 0.03 & \pm 0.08 & \\
\hline \multirow{2}{*}{$28-30$} & $5.68^{\mathrm{b}}$ & $5.71^{\mathrm{ab}}$ & $5.83^{\mathrm{ab}}$ & $5.74^{\mathrm{ab}}$ & $5.81^{\mathrm{ab}}$ & $5.92^{\mathrm{ab}}$ & $5.95^{\mathrm{a}}$ & \multirow{2}{*}{0.25} \\
& \pm 0.13 & \pm 0.04 & \pm 0.07 & \pm 0.10 & \pm 0.09 & \pm 0.03 & \pm 0.10 & \\
\hline \multirow{2}{*}{$30-32$} & 5.75 & 5.73 & 5.76 & 5.78 & 5.81 & 5.94 & 5.94 & \multirow{2}{*}{$\mathrm{NS}$} \\
& \pm 0.10 & \pm 0.12 & \pm 0.11 & \pm 0.08 & \pm 0.06 & \pm 0.04 & \pm 0.05 & \\
\hline \multirow{2}{*}{$32-34$} & $5.65^{\mathrm{d}}$ & $5.75^{\mathrm{bcd}}$ & $5.91^{\mathrm{ab}}$ & $5.71^{\mathrm{cd}}$ & $5.85^{\mathrm{abc}}$ & $5.91^{\mathrm{ab}}$ & $5.95^{\mathrm{a}}$ & \multirow{2}{*}{0.19} \\
& \pm 0.07 & \pm 0.04 & \pm 0.08 & \pm 0.08 & \pm 0.04 & \pm 0.08 & \pm 0.05 & \\
\hline \multirow{2}{*}{$34-36$} & $5.56^{\mathrm{c}}$ & $5.65^{\mathrm{bc}}$ & $5.76^{\mathrm{abc}}$ & $5.80^{\mathrm{abc}}$ & $5.89^{\mathrm{a}}$ & $5.94^{\mathrm{a}}$ & $5.93^{\mathrm{a}}$ & \multirow{2}{*}{0.25} \\
& \pm 0.08 & \pm 0.04 & \pm 0.08 & \pm 0.15 & \pm 0.10 & \pm 0.06 & \pm 0.07 & \\
\hline \multirow{2}{*}{$36-38$} & $5.42^{\mathrm{d}}$ & $5.60^{\mathrm{cd}}$ & $5.73^{\mathrm{bc}}$ & $5.68^{\mathrm{bc}}$ & $5.82^{\mathrm{ab}}$ & $5.86^{\mathrm{ab}}$ & $5.92^{\mathrm{a}}$ & \multirow{2}{*}{0.18} \\
& \pm 0.07 & \pm 0.05 & \pm 0.07 & \pm 0.08 & \pm 0.04 & \pm 0.07 & \pm 0.03 & \\
\hline \multirow{2}{*}{ Mean } & $5.59^{\mathrm{d}}$ & $5.65^{\mathrm{d}}$ & $5.75^{\mathrm{bc}}$ & $5.67^{\mathrm{cd}}$ & $5.78^{\mathrm{ab}}$ & $5.85^{\mathrm{a}}$ & $5.86^{\mathrm{a}}$ & \multirow{2}{*}{0.08} \\
& \pm 0.03 & \pm 0.03 & \pm 0.03 & \pm 0.04 & \pm 0.03 & \pm 0.03 & \pm 0.03 & \\
\hline
\end{tabular}

The mean values in same row with different superscripts differ significantly $(\mathrm{P}<0.05)$. 
Table.9 Mean values of egg shape index during progressive age (weeks) under different dietary treatments

\begin{tabular}{|l|c|c|c|c|c|c|c|c|}
\hline $\begin{array}{l}\text { Weeks/ } \\
\text { Treatment }\end{array}$ & $\mathbf{T}_{\mathbf{1}}$ & $\mathbf{T}_{\mathbf{2}}$ & $\mathbf{T}_{\mathbf{3}}$ & $\mathbf{T}_{\mathbf{4}}$ & $\mathbf{T}_{\mathbf{5}}$ & $\mathbf{T}_{\mathbf{6}}$ & $\mathbf{T}_{\mathbf{7}}$ & CD \\
\hline \multirow{2}{*}{$22-24$} & 69.90 & 73.48 & 72.18 & 74.82 & 71.48 & 71.07 & 72.91 & \multirow{2}{*}{ NS } \\
& \pm 1.31 & \pm 0.60 & \pm 0.58 & \pm 1.12 & \pm 1.18 & \pm 1.74 & \pm 1.25 & \\
\hline \multirow{2}{*}{$24-26$} & 73.11 & 70.57 & 72.42 & 71.78 & 72.04 & 71.67 & 71.63 & \multirow{2}{*}{ NS } \\
& \pm 1.46 & \pm 0.63 & \pm 1.07 & \pm 0.89 & \pm 0.52 & \pm 1.07 & \pm 1.76 & \\
\hline \multirow{2}{*}{$26-28$} & 73.20 & 72.63 & 73.01 & 72.58 & 73.88 & 72.98 & 73.76 & \multirow{2}{*}{ NS } \\
& \pm 1.05 & \pm 1.10 & \pm 0.43 & \pm 1.31 & \pm 2.18 & \pm 0.81 & \pm 2.92 & \\
\hline \multirow{2}{*}{$28-30$} & 70.12 & 72.42 & 71.23 & 72.63 & 72.39 & 72.32 & 71.45 & \multirow{2}{*}{ NS } \\
& \pm 2.35 & \pm 0.46 & \pm 0.94 & \pm 2.04 & \pm 1.48 & \pm 0.47 & \pm 3.02 & \\
\hline \multirow{2}{*}{$30-32$} & 72.11 & 73.51 & 72.69 & 72.21 & 72.33 & 71.25 & 71.10 & \multirow{2}{*}{ NS } \\
& \pm 1.10 & \pm 0.98 & \pm 1.21 & \pm 0.97 & \pm 1.27 & \pm 0.69 & \pm 0.84 & \\
\hline \multirow{2}{*}{$32-34$} & 73.16 & 72.05 & 71.77 & 72.41 & 72.00 & 72.40 & 73.18 & \multirow{2}{*}{ NS } \\
& \pm 1.10 & \pm 0.80 & \pm 0.46 & \pm 1.96 & \pm 0.81 & \pm 1.39 & \pm 1.13 & \\
\hline \multirow{2}{*}{$34-36$} & 72.79 & 73.76 & 73.36 & 69.72 & 71.24 & 72.13 & 71.37 & \multirow{2}{*}{ NS } \\
& \pm 1.71 & \pm 1.19 & \pm 1.19 & \pm 1.51 & \pm 1.14 & \pm 2.27 & \pm 0.87 & \\
\hline \multirow{2}{*}{$36-38$} & $75.30^{\mathrm{a}}$ & $72.93^{\mathrm{ab}}$ & $71.66^{\mathrm{b}}$ & $73.64^{\mathrm{ab}}$ & $72.89^{\mathrm{ab}}$ & $71.56^{\mathrm{b}}$ & $71.28^{\mathrm{b}}$ & \multirow{2}{*}{3.26} \\
& \pm 0.56 & \pm 0.83 & \pm 1.00 & \pm 0.81 & \pm 0.76 & \pm 2.00 & \pm 1.29 & \\
\hline \multirow{2}{*}{ Mean } & 72.46 & 72.60 & 72.50 & 72.48 & 72.28 & 71.92 & 72.09 & \multirow{2}{*}{ NS } \\
& \pm 0.53 & \pm 0.32 & \pm 0.32 & \pm 0.50 & \pm 0.42 & \pm 0.47 & \pm 0.61 & \\
\hline
\end{tabular}

The mean values in same row with different superscripts differ significantly $(\mathrm{P}<0.05)$. Economics of feeding linseed oil

Table.10 Average feed cost (Rs) per dozen egg production during progressive age (Weeks) under different dietary treatments

\begin{tabular}{|l|l|l|l|l|l|l|l|}
\hline $\begin{array}{l}\text { Weeks/ } \\
\text { Treatment }\end{array}$ & $\mathbf{T}_{\mathbf{1}}$ & $\mathbf{T}_{\mathbf{2}}$ & $\mathbf{T}_{\mathbf{3}}$ & $\mathbf{T}_{\mathbf{4}}$ & $\mathbf{T}_{\mathbf{5}}$ & $\mathbf{T}_{\mathbf{6}}$ & $\mathbf{T}_{\mathbf{7}}$ \\
\hline $22-24$ & 45.54 & 47.44 & 50.72 & 43.01 & 39.61 & 50.65 & 60.89 \\
\hline $24-26$ & 41.34 & 54.72 & 49.55 & 47.66 & 49.38 & 52.43 & 53.80 \\
\hline $26-28$ & 48.17 & 60.59 & 50.52 & 54.47 & 58.85 & 49.52 & 52.97 \\
\hline $28-30$ & 53.20 & 57.59 & 62.93 & 53.47 & 57.92 & 58.46 & 54.51 \\
\hline $30-32$ & 54.84 & 57.11 & 62.35 & 52.99 & 60.35 & 62.86 & 62.42 \\
\hline $32-34$ & 57.61 & 57.08 & 56.11 & 53.62 & 61.86 & 63.13 & 62.24 \\
\hline $34-36$ & 58.52 & 57.17 & 60.94 & 53.95 & 59.29 & 58.03 & 63.51 \\
\hline $36-38$ & 59.48 & 62.00 & 60.16 & 54.03 & 64.55 & 65.54 & 62.81 \\
\hline Mean & 52.33 & 56.71 & 56.66 & 51.65 & 56.47 & 64.82 & 59.14 \\
\hline Profit/loss & 0 & -4.38 & -4.33 & 0.68 & -4.14 & -12.49 & -6.81 \\
\hline
\end{tabular}


Table.11 Mean values of different fatty acids \% in egg yolk of layers under different treatments

\begin{tabular}{|c|c|c|c|c|c|c|c|}
\hline Treatment & $\begin{array}{c}\text { C:16 } \\
\text { (palmitic } \\
\text { acid) }\end{array}$ & $\begin{array}{c}\text { C:18 } \\
\text { (Stearic } \\
\text { Acid) }\end{array}$ & $\begin{array}{l}\text { C18:1 } \\
\text { (Oleic } \\
\text { acid) }\end{array}$ & $\begin{array}{c}\text { C18:2 } \\
\text { (Linoleic } \\
\text { acid) }\end{array}$ & $\begin{array}{c}\text { C18:3 } \\
\text { (Linolenic } \\
\text { acid) }\end{array}$ & $\begin{array}{c}\text { C20:4 } \\
\text { (Archidonic } \\
\text { acid }\end{array}$ & n6:n3 \\
\hline T1 & $\begin{array}{c}34.21^{\mathrm{a}} \pm 0.0 \\
1\end{array}$ & $\begin{array}{c}13.29^{\mathrm{a}} \pm 0.0 \\
1\end{array}$ & $\begin{array}{c}38.12^{\mathrm{a}} \pm 0.0 \\
1\end{array}$ & $\begin{array}{c}12.13^{\mathrm{g}} \pm 0.0 \\
1\end{array}$ & $\begin{array}{c}1 \\
52^{\mathrm{g}} \pm 0.01\end{array}$ & $0.74^{\mathrm{f}} \pm 0.01$ & 8.46 \\
\hline T2 & $\begin{array}{c}33.50^{\mathrm{b}} \pm 0.0 \\
1\end{array}$ & $\begin{array}{c}12.17^{\mathrm{b}} \pm 0.0 \\
1\end{array}$ & $\begin{array}{c}37.40^{\mathrm{b}} \pm 0.0 \\
1\end{array}$ & $\begin{array}{c}13.23^{\mathrm{f}} \pm 0.0 \\
1\end{array}$ & $2.71^{\mathrm{f}} \pm 0.01$ & $0.89^{\mathrm{e}} \pm 0.01$ & 5.21 \\
\hline T3 & $\begin{array}{c}32.00^{\mathrm{c}} \pm 0.0 \\
4\end{array}$ & $\begin{array}{c}11.05^{\mathrm{c}} \pm 0.0 \\
4\end{array}$ & $\begin{array}{c}37.45^{\mathrm{c}} \pm 0.0 \\
2\end{array}$ & $\begin{array}{c}14.45^{\mathrm{e}} \pm 0.0 \\
2\end{array}$ & $4.10^{\mathrm{e}} \pm 0.01$ & $0.93^{\mathrm{d}} \pm 0.01$ & 3.75 \\
\hline T4 & $\begin{array}{c}31.06^{\mathrm{d}} \pm 0.0 \\
1\end{array}$ & $\begin{array}{c}11.00^{\mathrm{c}} \pm 0.0 \\
1\end{array}$ & $\begin{array}{c}37.09^{\mathrm{d}} \pm 0.0 \\
1\end{array}$ & $\begin{array}{c}14.61^{\mathrm{d}} \pm 0.0 \\
1\end{array}$ & $5.26^{\mathrm{d}} \pm 0.01$ & $0.98^{\mathrm{c}} \pm 0.01$ & 2.96 \\
\hline T5 & $\begin{array}{c}30.25^{\mathrm{e}} \pm 0.0 \\
2\end{array}$ & $\begin{array}{c}10.16^{\mathrm{d}} \pm 0.0 \\
2\end{array}$ & $\begin{array}{c}36.46^{\mathrm{e}} \pm 0.0 \\
1\end{array}$ & $\begin{array}{c}15.05^{\mathrm{c}} \pm 0.0 \\
1\end{array}$ & $7.08^{\mathrm{c}} \pm 0.01$ & $1.00^{\mathrm{c}} \pm 0.01$ & 2.26 \\
\hline T6 & $\begin{array}{c}29.30^{\mathrm{f}} \pm 0.0 \\
1\end{array}$ & $9.03^{\mathrm{e}} \pm 0.01$ & $\begin{array}{c}36.56^{\mathrm{f}} \pm 0.0 \\
1\end{array}$ & $\begin{array}{c}15.68^{\mathrm{b}} \pm 0.0 \\
1\end{array}$ & $8.35^{\mathrm{b}} \pm 0.02$ & $1.08^{\mathrm{b}} \pm 0.02$ & 2.00 \\
\hline T7 & $\begin{array}{c}28.35^{\mathrm{g}} \pm 0.0 \\
4\end{array}$ & $8.19^{\mathrm{f}} \pm 0.04$ & $\begin{array}{c}36.10^{\mathrm{g}} \pm 0.0 \\
1\end{array}$ & $\begin{array}{c}16.12^{\mathrm{a}} \pm 0.0 \\
1\end{array}$ & $9.79^{\mathrm{a}} \pm 0.01$ & $1.45^{\mathrm{a}} \pm 0.01$ & 1.79 \\
\hline CD & 0.06 & 0.06 & 0.04 & 0.04 & 0.04 & 0.04 & \\
\hline
\end{tabular}

The mean values in same column with different superscripts differ significantly $(\mathrm{P}<0.05)$.

\section{Egg width and egg length}

The collective mean values (22-38 weeks) of egg width and egg length were $4.04,4.10$, $4.15,4.10,4.17,4.20$ and $4.22 \mathrm{~cm}$ (Table 7); and 5.59, 5.65, 5.75, 5.67, 5.78, 5.85 and 5.86 $\mathrm{cm}$ (Table 8) in treatment groups $\mathrm{T}_{1}, \mathrm{~T}_{2}, \mathrm{~T}_{3}$, $\mathrm{T}_{4}, \mathrm{~T}_{5}, \mathrm{~T}_{6}$ and $\mathrm{T}_{7}$, respectively. The results depicted that there was significant $(\mathrm{P}<0.05)$ increase in egg width and egg length over the different experimental periods and maximum values were observed in the treatment group $\left(\mathrm{T}_{7}\right)$ of hens fed with highest level $(4 \%)$ of linseed oil during progressive weeks of age of birds. The mean values of egg width during $26^{\text {th }}, 32^{\text {nd }}$ and $38^{\text {th }}$ weeks and egg length in $32^{\text {nd }}$ weeks of age were found to be nonsignificant. With respect to the whole period, it was observed that there was significant $(\mathrm{P}<0.05)$ increase in both egg width and egg length under various linseed oil regimes $\left(\mathrm{T}_{2}\right.$, $\mathrm{T}_{3}, \mathrm{~T}_{4}, \mathrm{~T}_{5}, \mathrm{~T}_{6}$ and $\mathrm{T}_{7}$ ) as compared to the control $\left(\mathrm{T}_{1}\right)$ group. While, the maximum increase was observed with the highest level (4\%) of linseed oil in $\mathrm{T}_{7}$ treatment group, followed by the hens in treatment group $\mathrm{T}_{6}$ fed with $3.5 \%$ of linseed oil. Thus, the result findings clearly indicate that there is a notable increase in egg width and egg length with the increasing level of linseed oil in the ration of layer hens. Although, no literature was present in context of layer's egg length and egg width

\section{Egg shape index}

The cumulative average percent egg shape index was 72.46, 72.60, 72.50, 72.48, 72.28, 71.92 and 72.09 in treatment groups $\mathrm{T}_{1}, \mathrm{~T}_{2}$, $\mathrm{T}_{3}, \mathrm{~T}_{4}, \mathrm{~T}_{5}, \mathrm{~T}_{6}$ and $\mathrm{T}_{7}$, respectively (Table 9 ). The results of the study depicted that there were no significant differences among different dietary treatments during progressive weeks of age of hens as well as with respect to the period except in $38^{\text {th }}$ week. Thus, it can be concluded that feeding of different levels of linseed oil in the diet of laying hens did not affect egg shape index. Although, no literature was present in context of layer's egg shape index.

Feed costs (Rs.) in terms of per dozen egg production during progressive weeks of age are given in Table 10. The cumulative mean 
values of feed cost per dozen egg productions were Rs. 52.33, 56.71, 56.66, 51.65, 56.47, 64.82 and 59.14 in treatment groups $\mathrm{T}_{1}, \mathrm{~T}_{2}$, $\mathrm{T}_{3}, \mathrm{~T}_{4}, \mathrm{~T}_{5}, \mathrm{~T}_{6}$ and $\mathrm{T}_{7}$, respectively. The results showed that feed cost value for dozen egg production decrease in treatment groups $\mathrm{T}_{4}$ ( $2.5 \%$ linseed oil) in comparison to $\mathrm{T}_{1}$ (non supplemented maize based control diet). Thus, the result findings clearly indicate that highest net profit was obtained in hens of treatment group $\mathrm{T}_{4}$ only. Omar et al., (2014) investigated that Using 1\% Fish oil only or $1 \%$ Fish oil $+1 \%$ Linseed oil in laying hen diets improved the economical efficiency comparable to the control group. The best feed cost $/ \mathrm{kg}$ egg was recorded by the group fed diet $1 \%$ Fish oil $+1 \%$ Linseed oil.

\section{Fatty acid profile}

The mean values of palmitic acid (C: 16), in egg yolk were $34.21,33.50,32.00,31.06$, $30.25,29.30$ and 28.35 in treatment groups $\mathrm{T}_{1}, \mathrm{~T}_{2}, \mathrm{~T}_{3}, \mathrm{~T}_{4}, \mathrm{~T}_{5}, \mathrm{~T}_{6}$ and $\mathrm{T}_{7}$, respectively (Table 11). Results revealed that values of palmitic acid decrease significantly $(\mathrm{P}<0.05)$ with the increasing level of linseed oil. The mean values of Stearic acid (C: 18) were 13.29, 12.17, 11.05, 11.00. 10.16, 9.03 and 8.19 in treatment groups $\mathrm{T}_{1}, \mathrm{~T}_{2}, \mathrm{~T}_{3}, \mathrm{~T}_{4}, \mathrm{~T}_{5}, \mathrm{~T}_{6}$ and $\mathrm{T}_{7}$, respectively. Study depicted that stearic acid decreases significantly $(\mathrm{P}<0.05)$ with the increasing level of linseed oil. Thus saturated fatty acids (Palmitic and Stearic) decrease with increasing level of linseed oil. The mean values of Oleic acid (18:1) were $38.12,37.40,37.45,37.09,36.46,36.56$ and 36.10 for $\mathrm{T}_{1}, \mathrm{~T}_{2}, \mathrm{~T}_{3}, \mathrm{~T}_{4}, \mathrm{~T}_{5}, \mathrm{~T}_{6}$ and $\mathrm{T}_{7}$, respectively. The present study showed that there was significant $(\mathrm{P}<0.05)$ decrease in the level of oleic acid as the level of linseed oil increased. The mean values of Linoleic acid (C18:2) were 12.13, 13.23, 14.45, 14.61, $15.05,15.68$ and 16.12 in dietary treatments $\mathrm{T}_{1}, \mathrm{~T}_{2}, \mathrm{~T}_{3}, \mathrm{~T}_{4}, \mathrm{~T}_{5}, \mathrm{~T}_{6}$ and $\mathrm{T}_{7}$, respectively. Study depicted that Linoleic acid increased significantly $(\mathrm{p}<0.05)$ with the increasing level of linseed oil. The mean values of linolenic acid (18:3) were 1.52, 2.71, 4.10, 5.26, 7.08, 8.35 and 9.79 for $\mathrm{T}_{1}, \mathrm{~T}_{2}, \mathrm{~T}_{3}, \mathrm{~T}_{4}, \mathrm{~T}_{5}$, $\mathrm{T}_{6}$ and $\mathrm{T}_{7}$, respectively. The present study showed that there was significant $(\mathrm{P}<0.05)$ increase in the level of Linolenic acid as the level of linseed oil increased. The mean values of Arachidonic acid (C20:4) in egg yolk were $0.74,0.89,0.93,0.98,1.00,1.08$ and 1.45 in treatment groups $\mathrm{T}_{1}, \mathrm{~T}_{2}, \mathrm{~T}_{3}, \mathrm{~T}_{4}$, $\mathrm{T}_{5}, \mathrm{~T}_{6}$ and $\mathrm{T}_{7}$, respectively. Results reviled that Arachidonic acid (C20:4) increased significantly $(\mathrm{P}<0.05)$ with the increasing level of linseed oil. The present study showed that there was significant decrease in the n6: $\mathrm{n} 3$ ratio from $\mathrm{T}_{1}$ to $\mathrm{T}_{7}$. In agreement with present findings, Aymond and Elswyk, (1995), Ansari et al., (2006), Goncuglu and Ergun (2004), Van Elswyk (1997), Yalcyn et al., (2007), Galobart et al., (2001),. Grobas et al., (2001), Shapira et al., (2008), Souza et al., (2008), T. Sujatha and D. Narahari (2011), Kirubakaran A. et al., (2011), Oliveira et al., (2010) and Omar et al., (2014) reported that linseed oil supplementation in ration of layer increases PUFA and decreases n6:n3. This decrease in saturated fatty acid in egg yolk is due to the capability of hen to deposit added polyunsaturated fatty acid instead of saturated fatty acids.

It was concluded that supplementation of different levels of linseed oil in hens' diet decrease average feed cost per dozen egg production and significantly increase in hen day egg production omega-3 fatty acid and body weight. Decrease in n6: n3 ratio, which indicates increase in beneficial fatty acid. Whereas no effect of linseed oil supplementation on shape index.

\section{References}

A.O.A.C., 2007. Official Methods of Analysis. 19th ed. Association of 
Official Analytical Chemists, Gaitherburg, Madison.

Angelo, St. A. J., vercellotti, J. R., Legendre, M. G., Vinnett, C.H., Kuan, J. W. Jr., James, C. and Duppy, H. P. 1987. Chemical and instrumental analysis of warmed over flavor in beef. J. Food Sci. 52(5):1163-1166.

Ansari, R., Azarbayejani, A. Ansari, S. Asgari, S. and Gheisari, A. 2006. Production of egg enriched with omega3 fatty acids in laying hens. Arya. J. 1:242-246.

Augustyn, R., Barteczko, J. and Smulikowska, S. 2006. The effect of feeding regular or low $\alpha$-linolenic acid linseed on laying performance and total cholesterol content in eggs. J. Anim. Feed Sci. 15:103-106.

Aymond, W. M. and Van Elswyk, M. E. 1995. Yolk thiobarbituric acid reactive substances and n-3 fatty acids in response to whole and ground flaxseed. Poult. Sci. 74:1358-1364.

Aziza., A. E., Panda, A. K., Quezada, N. and. Cherian, G. 2013. Nutrient digestibility, egg quality, and fatty acid composition of brown laying hens fed camelina or flaxseed meal. J. Appl. Poult. Res. 22: 832-841.

Beynen, A. C. 2004. Fatty acid composition of eggs produced by hens fed diets containing groundnut, soybean or linseed. N. J. Anim. Sci. 52:3-10.

Bureau of Indian Standards, BIS. 2007. Poultry Feeds -specification. 5th review. IS: 1374-2007, Manak Bhawan, 9 Bahadurshah Zafar Marg, and New Delhi 110001.

Celebi, S. and Utlu, N. 2006. Influence of animal and vegetable oil in layer diets on performance and serum lipid profile. Int. J. Poult. Sci. 5:370-373.

Chu, T. K., and Kummerow, F. A. 1950. The deposition of linolenic acid in chickens fed linseed oil. Poultry Sci. 29:846-851.
Galobart, J., Barroeta, A. C., Baucells, M. D., Cortinas, L. and Guardiola, F. 2001. $\alpha-$ Tocopherol transfer efficiency and lipid oxidation in fresh and spray dried eggs enriched with $\omega 3$-polyunsaturated fatty acids. Poult. Sci. 80:1496-1505.

Goncuglu, E., and Ergun, A. 2004. The effect of flaxseed oil to egg quality, fatty acids and cholesterol content of egg yolk in laying hens. 22nd World Poultry Congress, Istanbul, Turkey.

Grobas, S., Mendez, J., Lazaros, R., Blas, C.D., Mateos, G.G. and De, B.C. 2001. Influence of source of fat added to diet on performance and fatty acid composition of egg yolks of two strains of laying hens. Poult. Sci. 80:11711179.

Kirubakaran, A., Narahari, D., Ezhil,Valavan, T., Sathish and Kumar, A. 2011. Effect of flaxseed,sardines, pearl millet, and holy basil leaves $\mathrm{n}$ production traits of layers and fatty acid composition of egg yolks. J. Poult sci. 90(1): 147-56.

Klatt, L. 1986. The lure of omega-3polyunsaturated fatty acids. Food Sci. Newsl. 16:1-4.

Klose, A. A., Mecchi, E. P., Behman, G. A., Lineweaver, H., Kratzer, F. $\mathrm{H}$ and Williams, D. 1952). Chemical characteristics of turkey carcass fat as a function of dietary fat. Poultry Sci. 31:354-359.

Kummerow, F. A., Hite J. and Kloxin, S. 1948. Fat rancidity in eviscerated poultry. The effect of variation in diet on the characteristics of the fat extracted from immature turkeys. Poultry Sci. 27:689-694.

Luddy, F. E., Barford, R. A., Herb, S. F. and Magdman, P. 1968. A rapid and quantitative procedure for preparation of methyl esters of butteroil and other fats. J. American Oil Chemists Society. 45(8):549-552

Novak, C., and Scheideler S. E. 2001. Long- 
term effects of feeding flaxseed based diets. 1. Egg production parameters, components, and eggshell quality in two strains of laying hens. Poult. Sci. 80:1480-1489.

Oliveira, D. D., Baiao, N. C., Cançado, S. V., Grimaldi, R., Souza, M. R., Lara, L. J. and Lana, A. M. 2010 Effects of lipid sources in the diet of laying hens on the fatty acid profiles of egg yolks. Poult Sci. 89(11):2484-90.

Omar Amal.S., Nehad, Ramadan, A., Bahakaim, A. S. A., Sahar, Osman, M. H. and Abdel Malak, N. Y. 2014. Effect of using different levels of fish oil, linseed oil and their combination in layer diet on effect of omega 3 enrichment. J. Animal and Poultry Prod. 5(12): 759 - 774

Sarica, M and C. Erensayin, 2009. Poultry Products. In: Turkoglum., M. Sarica: Poultry Science. Bey-Ofset, Ankara, Turkey. Pp. 89-138.

Shapira, N., Weill, P. and Loewenbach, R. 2008. Egg fortification with n-3 polyunsaturated fatty acids (PUFA): Nutritional benefits versus high $n-6$ PUFA western diets, and consumer acceptance. I. M. A. J. 10: 262-265

Snedecor, G.W. and Cochran, W.G. 1994.
Statistical methods. $6^{\text {th }}$ edition. The Iowa State University Press, Ames, Iowa, USA.

Souza, J. G., Costa, F. P. G., Queiroga, R. C. R. E., Silva, J. H. V., Schuler, A. R. P. and Goulart, C. C. 2008. Fatty acid profile of eggs of seamy-heavy layers fed feed containing linseed oil. Revista Brasileira de Ciencia Avicola. 10(1): 1590-1599.

Sujatha, T. and Narahari, D. 2011. Effect of designer diets on egg yolk composition of white leghorn hens. J. food sci. Techno. 48(4): 494-497.

Svedova M., Vasko, L., Trebunova, A., Kastel, R., Tuckova, M. and Certik, M. 2008. Influence of linseed and fish oil on metabolic and immunological indicators of laying hens. Acta Vet. Brno. 77: 3944.

Van Elswyk, M. E. 1997b. Nutritional and physiological effects of flax seed in diets for laying fowl. World's Poult. Sci. J. 53: 253-264.

Yalcyn, H., Ünal, M. K. and Basmacyoolu, H. 2007. The fatty acid and cholesterol composition of enriched egg yolk lipids obtained by modifying hens' diets with fish oil and flaxseed. Grasas Y. Aceites. 58(4): 372-378.

\section{How to cite this article:}

Promila, Nand Kishore, Sajjan Sihag, Jyoti Shunthwal, Rakesh Verma and Saurabh Baloda. 2017. Effect of Linseed Oil Supplementation on Hen Day Egg Production, Body Weight, Egg Shape Index, Economics and Egg Quality in Layers. Int.J.Curr.Microbiol.App.Sci. 6(11): 20052016. doi: https://doi.org/10.20546/ijcmas.2017.611.239 